\title{
When giving some away makes sense to jump-start the diffusion process
}

\author{
Donald R. Lehmann • Mercedes Esteban-Bravo
}

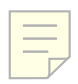

\begin{abstract}
This paper uses an analytical model to examine when it makes sense to provide incentives to innovators to adopt a new product. The model allows for separate segments of innovators and imitators, each of which follows a Bass-type diffusion process. Interestingly "seeding" the market is optimal for a limited range of situations and these do not appear to include those where there is a downturn in sales (chasm) as sales move from the first to the second segment.
\end{abstract}

Research has frequently identified different segments of adopters of new products. Categorizations include innovators vs imitators (Bass, 1969; Rogers, 1995; Mahahan et al., 1990; Im et al., 2003), technophiles vs "normal" people, and business vs consumer users. Further, considerable effort has gone into studying the influence of members of the first group on the second.

This paper focuses on when, if ever, it makes sense for a manufacture of a new product to "seed" the market by subsidizing a few early adopters to speed the adoption process. The paper builds on earlier work by Kalish and Lilien (1983) which focused on the impact of widely available government subsidies on the adoption of socially desirable innovations (i.e. alternative energy sources) as well as the work of Jain et al. (1995). Unlike that work, we concentrate on providing subsidies (here free goods) to selective individuals in the context of a model which allows for separate segments of innovators and imitators and nests the standard Bass (1969) model.

D R Lehmann $(\bowtie)$

Columbia University Graduate School of Business, 3022 Broadway, Uris Hall 507, New York,

NY 10027

e-mail: Drl2@columbia edu

M Esteban-Bravo

Universidad Carlos III de Madrid, C/ Mdrid, 126, 28903 Getafe Madrid Spain

e-mail: mesteban@emp uc3m es 


\section{Background}

The diffusion of new products is one of the most widely studied topics in marketing (Hauser et al., 2004). A large percentage of this work is based on the Bass (1969) model. This model parsimoniously describes S-shaped diffusion patterns by assuming a single diffusion pattern with separate coefficients capturing innovation and imitation tendencies.

Research focused on multiple diffusion processes has appeared in various forms. For example, Norton and Bass (1987) studied the adoption of successive generations of technology. Closer to the current problem, several researchers have looked at diffusion across countries, i.e. different populations (Dekimpe et al., 2000; Gatignon et al., 1989; Kalish et al., 1995; Putsis and Sen, 2001; Putsis et al., 1997; Takada and Jain, 1991; Tellis et al., 2003). In contrast to these papers, this paper looks at different segments within the same geographic area and time period. It does so using a latent class approach, rather than capturing heterogeneity within a single population (e.g., Bemmaor and Lee, 2002). Perhaps the closest work is that of Jain et al. (1995) which examined optimal sampling in the context of a single population and suggested it tends to be about $5 \%$ of the population for durables.

A related area of work concerns the impact adoption by others has on the value of a good, aka network externalities (cf Naik et al., 2003). Much of this work focuses on the implications of network effects on company behavior and the emergence of industry standards (e.g., Arthur, 1989; Farrell and Saloner, 1985; Gupta et al., 1999; Katz and Shapiro, 1985). For example, Sun et al. (2004) consider four strategies for innovators in a market with network effects. Other work centers on the notion of an information cascade (e.g., Bikhchandani et al., 1998; Golder and Tellis, 2004).

Some work explicitly considers two segments. Goldenberg et al., (2002) used cellular automata to simulate diffusion in two segments and found it can produce a "saddle" (a slow down in and then resumption of growth) in the diffusion pattern. Similarly Muller and Yogev (2004) studied 35 electronics markets and found empirical evidence for a saddle in 26 of them. In addition, Van den Bulte and Joshi (2005) built a two segment model and showed that for 33 cases it fits better than single population models. We use a similar model in this paper as the basis for our analytical results.

Other work has focused on the lead time until a product "takes-off" (Golder and Tellis, 1997; Kohli et al., 1999). Although this work does not explicitly consider two segments, the pattern established is consistent with a delay in time before a product enters a broader (mass) market.

The focus on firm behavior (rather than describing or predicting customer behavior) has a long history. Horsky and Simon (1983) incorporated the impact of advertising on diffusion, Robinson and Lakhani (1975) considered price, and Kalish and Lilien (1983) examined the role of price subsidies. Mahajan and Muller (1998) examined when it is optimal to target the mass market rather than innovators using a two segment model of pure innovators and pure imitators. This paper extends that work by considering the benefits of a subsidy to innovators for the purpose of speeding adoption in the second (larger) segment when the two segments have a propensity to both innovate and imitate.

This paper first describes when it is profitable to "seed" a diffusion process by giving away a product in a two segment world. The impact of varying model parameters on the 
optimal subsidy and profit is examined using numerical methods. The paper concludes with discussion and suggestions for future research.

\section{A two segment model}

The model assumes there are two segments: innovators where imitation is only due to adoptions within this group (i.e. B to B adoption only depends on adoption by other business) and imitators who are influenced by adoption in both groups.

The model

For both groups we assume adoption follows the Bass (1969) model in discrete time. We assume that $m$ is the total market potential and $\theta$ is the proportion of first group potential to total market potential. Therefore, $m_{1}=\theta m$ and $m_{2}=(1-\theta) m$. Similar to the extended Bass model (Bass et al., 1994) and the models of Robinson and Lakhani (1975) and Kalish and Lilien (1983), we assume price has a multiplicative impact on the hazard function. In the first segment the basic hazard function (probability of purchasing) is given by:

$$
H_{1}(t)=\left(p_{1}+q_{1} \frac{F_{1}(t)}{m_{1}}\right)
$$

and sales are $S_{1}(t)=H_{1}(t)\left(m_{1}-F_{1}(t)\right)$, where $F_{1}(t)$ is the cumulative adoption at time $t$. In the second segment the hazard rate is:

$$
H_{2}(t)=\left(p_{2}+q_{12} \frac{F_{1}(t)}{m_{1}}+q_{2} \frac{F_{2}(t)}{m_{2}}\right)
$$

and sales are $S_{2}(t)=H_{2}(t)\left(m_{2}-F_{2}(t)\right)$, where $F_{2}(t)$ is the cumulative adoption at time $t$. Here $q_{12}$ represents the effect of adoption by the first segment on the second and $q_{2}$ the imitation effect within the second segment. While not a model requirement, we would generally expect $q_{2}>q_{12}$, i.e. imitators are more influenced by the adoption of other imitators than adoption by innovators. The model has several special cases.

1. $\theta=1$ (or 0 ) The model reduces to a single population Bass model.

2. $q_{12}=0$ In this case, the two segments operate independently from each other, i.e. adoption in one group has no influence on adoption in the other

3. $p_{2}=0$ In this case, imitators only adopt through the influence of others.

4. $q_{1}=0$ Here the innovator segment strictly innovates, i.e. is uninfluenced by the actions of others.

A priori, we expect the first segment to consist of innovators and early adopters in the Rogers (1983) framework, thus representing about $16 \%$ of the total market. Following Muller and Yogev (2004), Goldenberg et al. (2002) and Van den Bulte and Joshi (2005), we examine the special case where $p_{2}=0$. This is consistent with behavioral descriptions of late majority and laggards as non-innovative (i.e. $p=0$ ).

Diffusion clearly can be impacted by marketing actions. Here we consider the impact of price. By using exp ( $-b_{1}$ price) as a multiple of the hazard rate, we have 
zero sales at an infinitive price and the base hazard rate sales at zero price. Specifically, taking into account the impact of prices, the first segment sales are given by

$$
\begin{aligned}
S_{1}(t) & =H_{1}(t)\left(m_{1}-F_{1}(t)\right) \exp \left(-b_{1} \operatorname{pr}(t)\right) \\
& =\left(p_{1}+q_{1} \frac{F_{1}(t)}{m_{1}}\right)\left(m_{1}-F_{1}(t)\right) \exp \left(-b_{1} \operatorname{pr}(t)\right)
\end{aligned}
$$

and the second segment sales by

$$
S_{2}(t)=\left(p_{2}+q_{12} \frac{F_{1}(t)}{m_{1}}+q_{2} \frac{F_{2}(t)}{m_{2}}\right)\left(m_{2}-F_{2}(t)\right) \exp \left(-b_{2} \operatorname{pr}(t)\right)
$$

where $\operatorname{pr}(t)$ denote the price at time $t$. We allow price to vary over time and investigate how it should be set in order to optimize profits.

The role of subsidies

One way to encourage sales is to subsidize adoption in the first segment (we consider subsidies to the second segment later) by giving the product to some or all members of the first segment, in effect "seeding" the population. Examples include provision of products to celebrities or key influentials whose behavior is then copied by the mass market.

Specifically, we allow the manager to subsidize $d$ members of the first segment (up to a maximum of $m_{1}$, the market potential) at a variable cost of $f$ per customer. (In the case of give-aways, $f$ becomes variable cost plus any delivery, etc. expenses). Note this differs from a price (promotion) subsidy available to the entire population as analyzed by Kalish and Lilien (1983). We assume the manager can identify who is in the innovator segment but not who within this segment will buy first (i.e. $p_{1}$ is constant across the group). When samples are given in the first period to the first segment, the sales of the first segment in the first period are thus

$$
S_{1}(1)=p_{1}\left(m_{1}-d\right) \exp \left(-b_{1} \operatorname{pr}(1)\right)
$$

and the total adoption is

$$
F_{1}(1)=p_{1}\left(m_{1}-d\right) \exp \left(-b_{1} \operatorname{pr}(1)\right)+d
$$

Thus give-aways decrease sales in the first period and increase them in subsequent periods.

Variable costs

We allow variable costs $(v c(t))$ to vary over time. Generally we expect them to decrease as experience curve effects manifest themselves. As we later show, the optimal path for price depends, unsurprisingly, on the pattern of variable costs over time. For simplicity, 
and since including them does not alter the character of the results, we assume fixed costs are zero (e.g., sunk).

The existence of a saddle

Since the work by Moore (1999), the notions of crossing the chasm and a saddle have been widely discussed if infrequently formally tested. We examined numerical simulations to see when a saddle in the total sales curve appears using the metaanalysis of Sultan et al. (1990) to inform the results. Specifically, we assumed the initial (innovator) segment had relatively large coefficients of innovation and imitation. We also assumed the first group was a relatively small fraction of the total potential market.

Interestingly, saddles only appear when the crossover imitation effect between the two segments $\left(q_{12}\right)$ is low, the first segment peaks early ( $p_{1}$ is fairly large), and the size of the first segment $(\theta)$ is moderate. When $\theta$ is small, the impact of the first group on total sales is minimal; when $\theta$ is large, the impact of the second is small. In either case, a saddle does not appear.

A saddle does appear in conditions suggested by the literature. For example, if the first segment has $p_{1}=.03$ and $q_{1}=.30$ (the averages reported by Sultan et al. (1990)), the second segment has $p_{2}=.0001, q_{12}=0.01$, and $q_{2}=0.10$ (i.e. diffusion is slower in the second group) and $\theta=.15$, a clear saddle emerges. In general the results suggest a saddle will occur when a product is complicated and/or not compatible with current ones (leading to a low $p_{2}$ ) or there is a benefit to waiting such as network externality (low $q_{2}$ ), consistent with Van den Bulte and Joshi (2005).

\section{Optimal behavior}

We focus on a three period model. Clearly if there is an infinite time horizon and a zero discount rate, subsidizing early adopters merely reduces sales. However, money has time value meaning that results in future periods have less and less value. In addition, for many products subsequent entry by competitors drives profit margins down toward pure competition levels. There is also the risk of technological obsolescence whereby a superior new technology captures the market from an older one. These issues are especially relevant for smaller firms and start-ups who have limited resources and, often, limited interest in operating in the long run (vs., say, selling out). As a consequence, it is reasonable to argue that a limited horizon model is applicable to many real situations. (If one wanted to capture, say, the first nine years of a diffusion process, they could consider three three-year periods, with the main impact being that the values of $p$ and $q$ would differ from those in an annual model).

Using three periods allows for an adoption by someone in the first segment in period one to be imitated by someone in the second segment in period two who then influences further adoption in the second segment in the third period. This allows us to capture the nature of the results while remaining reasonably tractable. Since most profits are often made early in the product life cycle, this captures the most critical period for the firm. Because subsidizing someone in the first segment in the first period dominates subsidizing them in subsequent periods in terms of their effect on adoption by others, we only consider subsidies in the initial period. 
Let:

$$
\begin{aligned}
\beta & =\text { the discount factor i.e. } \frac{1}{1+r} \text { where } r \text { is the discount rate, } \\
\operatorname{pr}(t) & =\text { unit price at time } t, \\
v c(t) & =\text { unit cost at time } t, \\
d & =\text { number subsidized, } \\
f & =\text { cost of a single subsidy. }
\end{aligned}
$$

The firm faces the problem of maximizing profit with respect to the number subsidized in period 1 and price in the three periods:

$$
\max \sum_{t=1}^{3} \beta^{t-1}(p r(t)-v c(t))\left(S_{1}(t)+S_{2}(t)\right)-d f,
$$

subject to the following constraints:

$$
\begin{aligned}
S_{1}(1)= & p_{1}\left(m_{1}-d\right) \exp \left(-b_{1} \operatorname{pr}(1)\right), \\
S_{2}(1)= & p_{2} m_{2} \exp \left(-b_{2} \operatorname{pr}(1)\right), \\
S_{1}(2)= & {\left[p_{1} m_{1}+\left(q_{1}-p_{1}\right)\left(S_{1}(1)+d\right)-\frac{q_{1}}{m_{1}}\left(S_{1}(1)+d\right)^{2}\right] \exp \left(-b_{1} \operatorname{pr}(2)\right), } \\
S_{2}(2)= & {\left[p_{2} m_{2}+\frac{q_{12}}{m_{1}} m_{2}\left(S_{1}(1)+d\right)+\left(q_{2}-p_{2}\right) S_{2}(1)\right.} \\
& \left.-\frac{q_{12}}{m_{1}}\left(S_{1}(1)+d\right) S_{2}(1)-\frac{q_{2}}{m_{2}} S_{2}(1)^{2}\right] \exp \left(-b_{2} \operatorname{pr}(2)\right), \\
S_{1}(3)= & {\left[p_{1} m_{1}+\left(q_{1}-p_{1}\right)\left(S_{1}(1)+S_{1}(2)+d\right)-\frac{q_{1}}{m_{1}}\left(S_{1}(1)+S_{1}(2)+d\right)^{2}\right] } \\
& \exp \left(-b_{1} \operatorname{pr}(3)\right), \\
S_{2}(3)= & {\left[p_{2} m_{2}+\frac{q_{12}}{m_{1}} m_{2}\left(S_{1}(1)+S_{1}(2)+d\right)+\left(q_{2}-p_{2}\right)\left(S_{2}(1)+S_{2}(2)\right)\right.} \\
& \left.\quad-\frac{q_{12}}{m_{1}}\left(S_{1}(1)+S_{1}(2)+d\right)\left(S_{2}(1)+S_{2}(2)\right)-\frac{q_{2}}{m_{2}}\left(S_{2}(1)+S_{2}(2)\right)^{2}\right] \\
& \quad \exp \left(-b_{2} \operatorname{pr}(3)\right) .
\end{aligned}
$$

Substituting the constraints into the firm's objective function leads to a notationally cumbersome problem for which numerical resolution typically is computationally expensive, in both time and memory requirements. A standard approach to solve largedimensional optimization problems is to exploit the special features of the problem. In this case, we reduce the computational cost in terms of function evaluations and number of iterations, re-writing the unconstrained formulation as a constrained problem which treat the sales equations as constraints. Both formulations are equivalent, but the 
constrained one is less costly to implement . The Lagrangian is defined by

$L(d, \operatorname{pr}(1), \operatorname{pr}(2), \operatorname{pr}(3), \lambda)$

$$
\begin{aligned}
= & \sum_{t=1}^{3} \beta^{t-1}(p r(t)-v c(t))\left(S_{1}(t)+S_{2}(t)\right)-d \times f \\
+ & \lambda_{1}\left[S_{1}(1)-p_{1}\left(m_{1}-d\right) \exp \left(-b_{1} p r(1)\right)\right] \\
+ & \lambda_{2}\left[S_{2}(1)-p_{2} m_{2} \exp \left(-b_{2} \operatorname{pr}(1)\right)\right] \\
+ & \lambda_{3}\left[S_{1}(2)-\left[p_{1} m_{1}+\left(q_{1}-p_{1}\right)\left(S_{1}(1)+d\right)-\frac{q_{1}}{m_{1}}\left(S_{1}(1)+d\right)^{2}\right]\right. \\
& \left.\quad \exp \left(-b_{1} \operatorname{pr}(2)\right)\right] \\
+ & \lambda_{4}\left[S_{2}(2)-\left[p_{2} m_{2}+\frac{q_{12}}{m_{1}} m_{2}\left(S_{1}(1)+d\right)+\left(q_{3}-p_{2}\right) S_{2}(1)\right.\right. \\
- & \left.\left.\frac{q_{12}}{m_{1}}\left(S_{1}(1)+d\right) S_{2}(1)-\frac{q_{2}}{m_{2}} S_{2}(1)^{2}\right] \exp \left(-b_{2} p r(2)\right)\right] \\
+ & \lambda_{5}\left[S_{1}(3)-\left[p_{1} m_{1}+\left(q_{1}-p_{1}\right)\left(S_{1}(1)+S_{1}(2)+d\right)\right.\right. \\
& \left.\left.-\frac{q_{1}}{m_{1}}\left(S_{1}(1)+S_{1}(2)+d\right)^{2}\right] \exp \left(-b_{1} \operatorname{pr}(3)\right)\right] \\
+ & \lambda_{6}\left[S_{2}(3)-\left[p_{2} m_{2}+\frac{q_{12}}{m_{1}} m_{2}\left(S_{1}(1)+S_{1}(2)+d\right)+\left(q_{2}-p_{2}\right)\left(S_{2}(1)+S_{2}(2)\right)\right.\right. \\
& \left.-\frac{q_{12}}{m_{1}}\left(S_{1}(1)+S_{1}(2)+d\right)\left(S_{2}(1)+S_{2}(2)\right)-\frac{q_{2}}{m_{2}}\left(S_{2}(1)+S_{2}(2)\right)^{2}\right] \\
& \left.\exp \left(-b_{2} \operatorname{pr}(3)\right)\right] .
\end{aligned}
$$

The Lagrange multipliers facilitate the characterization of optimal solutions, and also provide sensitivity information, quantifying the first-order variation in the optimal profit caused by variations in sales. The Lagrange multipliers $\lambda_{1}, \lambda_{2}, \lambda_{3}, \lambda_{4}, \lambda_{5}, \lambda_{6}$ at the optimum represent the value of one additional sale in each of the three periods $\left(\lambda_{1}, \lambda_{3}, \lambda_{5}\right.$ for the first segment and $\lambda_{2}, \lambda_{4}, \lambda_{6}$ for the second segment). As is logical, the value of one additional sale decreases over time $\left(\lambda_{1}>\lambda_{3}>\lambda_{5}\right.$ and $\left.\lambda_{2}>\lambda_{4}>\lambda_{6}\right)$ and is equal and positive in both segments in the last period $\left(\lambda_{5}=\lambda_{6}\right)$. Further, they are all positive, consistent with both the time value of money and the greater cumulative influence impact of earlier adoption.

Numerical results

We initially calculated the optimal values of the decision variables for several sets of parameters. As a base case, we assumed an arbitrary total market size of 5,000. We used coefficients of innovation of 0.05 and 0.01 and coefficients of imitation of 0.6 
Table 1 Parameters' used in the analyses

The first value represents the base case

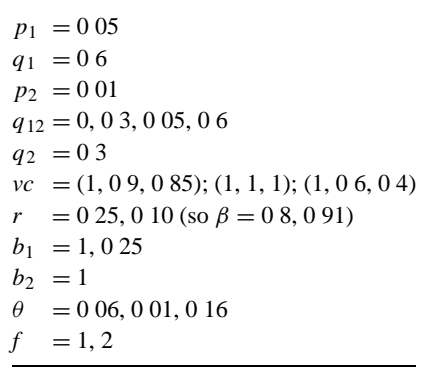

and 0.3 for the innovator and imitator segments respectively to reflect the range found in Sultan et al. (1990). For the coefficient of imitation for the second segment based on adopters in the first segment $\left(q_{12}\right)$ we initially used .30 . We assumed innovators were $6 \%$ of the population. We also assumed the variable cost was 1 and decreased to 90 and $85 \%$ of the first period's level over the three periods.

To capture the effect of price, we initially set $b_{1}$, and $b_{2}$ to 1 . This implies a price elasticity of -1 , less than the average of -1.76 reported by Tellis (1998) since new products are rarely bought primarily on a price basis. We also set $f=1$, i.e. the variable cost of the product, for the base case. The conditions used are summarized in Table 1.

For the base case, the optimal decision (Table 2) is to subsidize about 50\% (146 of 300 ) of the first segment by giving them the new product. Price declines somewhat but is essentially constant. Sales are limited in the first segment but substantial in the second although cumulative sales of 249 represent a small fraction $(6 \%)$ of the 4500 potential purchasers.

In order to estimate the sensitivity of the optimal solution to the various model parameters, we varied one at a time using the levels in Table 1. The number subsidized decreases as the imitation effect of the first group on the second $\left(q_{12}\right)$ goes down and reaches 0 at $q_{12}=.05$ and increases to $300(100 \%)$ when the first group has a strong impact on the second $\left(q_{12}=.6\right)$. A lower discount rate leads to more subsides as do more rapidly decreasing variable costs.

More interesting, as the size of the first segment $(\theta)$ moves toward zero, so does the number of subsidies. The number (and percent) subsidized decreases when $\theta$ becomes large. Thus subsidies are most beneficial when there is a small but not very small first segment. The size of the first segment also impacts the pricing pattern with higher and decreasing prices for a smaller $(\theta=0.01)$ and lower but increasing prices for a larger first segment $(\theta=0.16)$. More expensive subsidies lead to both a lower initial price and increasing prices across the three periods. When the variable cost is constant, optimal price increases over the three periods. If variable cost decreases substantially, so does price but not by as much in percentage terms. This suggests that in the absence of competition and scale economies, variable margins should increase over time. Interestingly, it does not appear to be worthwhile to subsidize customers in the first segment in those cases where a saddle occurs in the demand curve (e.g., for $\theta=0.16)$. In other words, it doesn't make sense to subsidize innovators in order to cross the chasm when the innovators and imitators operate separately. 


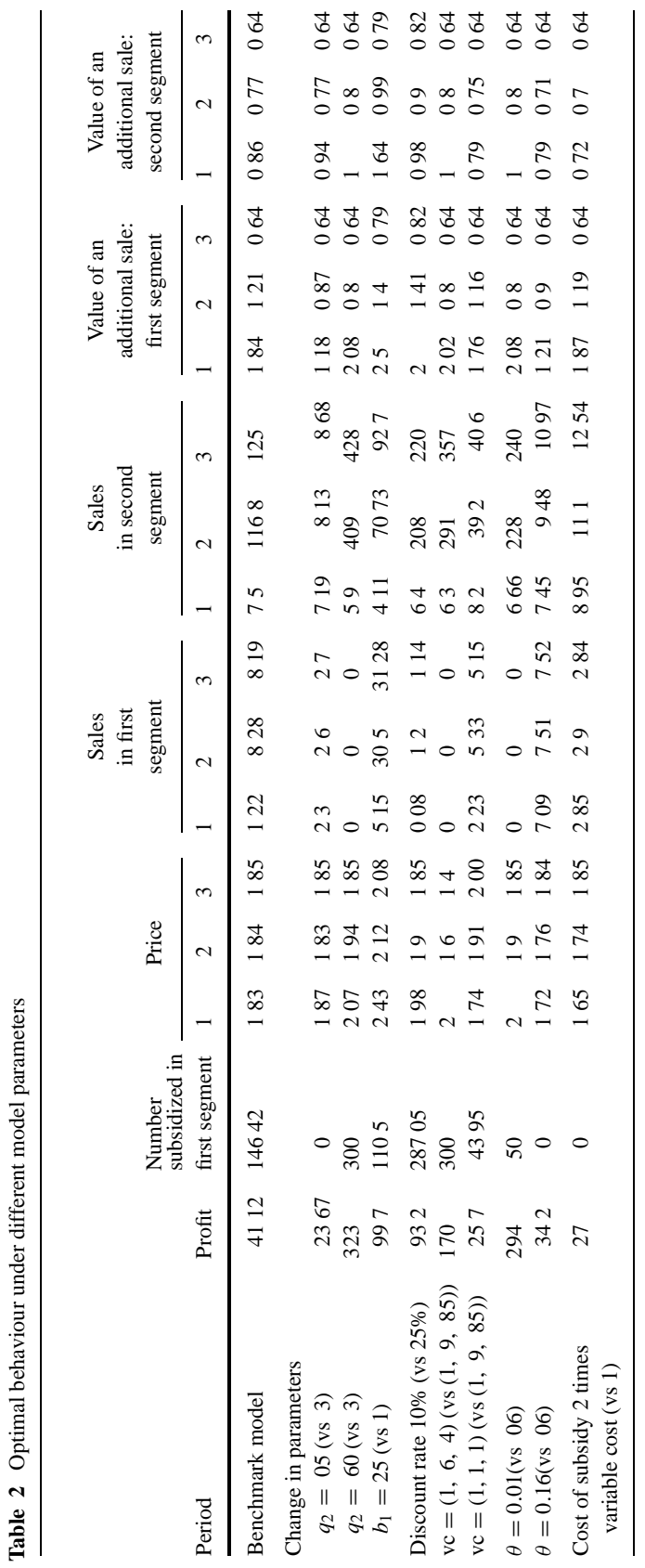


Table 3 Optimal percent to subsidize regression

\begin{tabular}{|c|c|c|c|c|c|}
\hline \multicolumn{3}{|c|}{ Segment 1} & \multicolumn{3}{|c|}{ Segment 2} \\
\hline Parameter & Coefficient & $t$ & Parameter & Coefficient & $t$ \\
\hline$\theta$ & 1043 & 1569 & $\theta$ & 022 & 695 \\
\hline$p_{1}$ & -087 & -559 & $p_{1}$ & 00018 & 165 \\
\hline$q_{1}$ & -084 & -530 & $p_{2}$ & -0038 & -331 \\
\hline$q_{12}$ & 254 & 2389 & $q_{12}$ & -0033 & -854 \\
\hline$\theta^{2}$ & -5215 & -1631 & $q_{2}$ & -0016 & -246 \\
\hline$p_{1}{ }^{2}$ & 082 & 387 & $\theta^{2}$ & -093 & -611 \\
\hline$q_{1}^{2}$ & 077 & 346 & $q_{12}^{2}$ & 0027 & 679 \\
\hline$q_{12}{ }^{2}$ & -188 & -1728 & $q_{2}{ }^{2}$ & 0025 & 294 \\
\hline
\end{tabular}

\section{Optimal behavior: Subsidies to both groups}

While the logic of the paper is based on the notion of subsidizing members of the first segment, obviously it is possible to subsidize members of the second segment as well. To deal with this situation, we created 368 scenarios representing different values of the parameters and numerically estimated optimal subsidies in both segments. To simplify the task, we assumed the variable cost was equal to the cost of subsidy at one ( $\mathrm{vc}=f=1$ ) and set the price to 2 (a $100 \%$ markup).

In order to interpret the results, we ran regressions of the optimal percent to subsidize in each segment against the model parameters. In order to allow for non-linearities we included both linear and quadratic terms. Table 3 presents the results for a reduced model which includes significant predictors.

Using the parameters from Table 3, we determined the values of each parameter which lead to maximum (or minimum) subsidy by taking derivates of the appropriate regression parameters and setting them equal to zero. In the first segment, the optimal percent to subsidize is largest when $\theta$ is close to $10 \%$, and smallest when $p_{1}$ and $q_{1}$ are close to 0.5 . The only other significant influence is $q_{12}$ the cross-population coefficient: subsidies are maximum for $q_{12}$ of 0.67 . Subsidies to the second segment are greatest for $\theta$ equal to about $12 \%$. They increase slightly in $p_{1}$ and decrease in $p_{2}$, as expected. Subsidies are smallest for $q_{12}$ of about .60 and $q_{2}$ of about .30 . Note that a $q_{2}$ of .30 is close to the mean found in prior research which may explain the lack of significant subsidies in most durable categories.

\section{Discussion}

This paper has demonstrated conditions under which it is optimal to "seed" a market consisting of two segments by giving away some of the product to innovators. Essentially this occurs when there is a small group of slow to adopt on their own customers who influence other customers.

Aside from the obvious implication that before deciding to subsidize managers should know their $p$ 's and $q$ 's (and $\theta$ ), a manager could use the model to consider strategies to improve adoption. For example, it may be possible to increase $q_{12}$ (i.e. WOM from innovators to imitators) through general publicity about early adopters 
or by viral marketing. In any event, subsidizing innovators should be more useful for cases where innovators (experts, opinion leaders) are most influential such as fashion goods or business technology.

More generally, a number of complications could be added to the situation. These include incorporating other decision variables such as advertising, incorporating a channel structure where the channel makes decisions about stocking levels, and allowing for subsequent competition. One can also expand the model to allow adoption in the second segment to influence adoption in the first. In addition, it would be useful to develop methods for estimating the model, especially when only aggregate data is available. Hopefully future research will deal with these and other issues.

\section{References}

Arthur, B W (1989) Competing technologies, increasing returns, and lock-in by historical events Economic Journal, 99(394), 116-131

Bass, FM , Krishnan, T V, \& Jain, D C (1994) Why the bass model fits without decision variables Marketing Science, 13(3), 203-223

Bass, FM (1969) A new product growth model for consumer durables Management Science, 15(5), 215-227

Bemmaor, A C , \& Lee, J (2002) The impact of heterogeneity and ill-conditioning on diffusion model parameter estimates Marketing Science, 2(2), 209-220

Bikhchandani, S, Hirschleifer, D , \& Welch, I (1998) Learning fro the behavior of others: conformity, fads, and informational cascades Journal of Economic Perspectives, 12(3), 151-170

Dekimpe, M G , Parker, PM , \& Sarvary, M (2000) Global diffusion of technological innovations: A coupled hazard approach Journal of Marketing Research, 37 (Issue Feb ), 47-59

Farrell, J , \& Saloner, G (1985) Standardization, compatibility, and innovation RAND Journal of Economics, 16(1), 70-83

Gatignon, H, Eliashberg, J , \& Robertson, T S (1989) Modeling multinational diffusion patterns: An efficient methodology Marketing Science, 8(3), 231-247

Goldenberg, J , Libai, B , \& Muller, E (2002) Riding the saddle: How cross-market communications can create a major slump in sales Journal of Marketing, 66, 1-16

Golder, PN , \& Tellis, G J (1997) Will it ever fly: modeling the takeoff of really new consumer durables Marketing Science, 16(3), 256-270

Golder, PN , \& Tellis, G J (2004) Growing, growing, gone: Cascades, diffusion, and turning points in the product life cycle Marketing Science, 23(2), 207-218

Gupta, S , Jain, D C , \& Sawhney, M S (1999) Modeling the evolution of markets with indirect network externalities: An application to digital television Marketing Science, 18(3), 396-416

Hauser, J , Tellis, G J , \& Griffin, A (2004) Research on innovation: a review and agenda for marketing working paper

Horsky, D , \& Simon, L S (1983) Advertising and the diffusion of new products Marketing Science, 2(1), $1-17$

Im, S , Bayus, B L , \& Mason, C H (2003) An empirical study of innate consumer innovativeness, personal characteristics, and new product adoption behavior Journal of the Academy of Marketing Science, 31(1), 61-73

Jain, D , Mahajan, V , \& Muller, E (1995) An approach for determining optimal product sampling for the diffusion of a new product Journal of Product Innovation Management, 12, 124-135

Kalish, S , \& Lilien, G L (1983) Optimal price subsidy policy for accelerating the diffusion of innovation Marketing Science, 2(4), 407-420

Kalish, S , Mahajan, V, \& Muller, E (1995) Waterfall and sprinkler new-product strategies in competitive global markets International Journal of Research in Marketing, 12 (Issue 2), 105-119

Katz, M L , \& Shapiro, C (1985) Network effects, competition, and compatibility American Economic Review, 75(3), 424-440

Kohli, R , Lehmann, D R , \& Pae, J H (1999) The extent and impact of incubation time in new porduct diffusion The Journal of Product Innovation Management, 16, 134-144 
Mahahan, V, Muller, E , \& Srivastava, R K (1990) Determination of adopter categories by using innovation diffusion models Journal of Marketing Research, 27, 37-50

Mahajan, V, \& Muller, E (1998) When is it worthwhile targeting the majority instead of the innovators in a new product launch? Journal of Marketing Research, 36(4), 488-495

Moore, G A (1999) Crossing the Chasm, 2nd (Ed) New York: Capstone

Muller, E , \& Yogev, G (2004) When does the majority become a majority? empirical analysis of the time at which main market adopters purchase the bulk of our sales working paper, Tel Aviv University

Nair, H , Chintagunta, P , \& Dube, J -P (2003) Empirical analysis of indirect network effects in the market for personal digital assistants working paper, University of Chicago

Norton, J , \& Bass, FM (1987) A diffusion theory model of adoption and substitution for successive generations of high-technology products Management Science, 33(9), 1069-1086

Putsis Jr, W P, \& Sen, S K (2001) International marketing and cross-country influences Marketing Science Conference Presentation Wiesbaden

Putsis Jr, W P, Balasubramaniam, S , Kaplan, E H , \& Sen, S K (1997) Mixing behavior in cross-country diffusion Marketing Science, 16(4), 354-369

Robinson, R , \& Lakhani, C (1975) Dynamic price models for new product planning Management Science, $21,1113-1122$

Rogers, E M (1983) The Diffusion of Innovation, 3rd (Ed) New York: The Free Press

Rogers, E M (1995) Diffusion of Innovations, 4th (Ed) New York: The Free Press

Sethuraman, R , \& Tellis, G (1991) An analysis of the tradeoff between advertising and price discounting Journal of Marketing Research, 28(2), 60-174

Sultan, F, Farley, J U , \& Lehmann, D R (1990) A meta-analysis of applications of diffusion models Journal of Marketing Research, 27, 70-77

Sun, B , Xie, J , \& Cao, H H (2004) Product strategy for innovators in markets with network effects Marketing Science, 23(2), 243-254

Takada, H , \& Jain, D (1991) Cross-national analysis of diffusion in durable goods in pacific rim countries Journal of Marketing, 55(2), 48-54

Tellis, G (1988) The price elasticity of selective demand: A meta-analysis of econometric models of sales Journal of Marketing Research, 25(4), 331-341

Tellis, G J , Stremersch, S , \& Yin, E (2003) The international takeoff of new products: economics, culture, and country innovativeness Marketing Science, 22(2), 88-208

Van den Bulte, C , \& Joshi, Y V (2005) New product diffusion with influentials and imitators, Working paper 